\title{
BULLYING EN ESTUDIANTES DE SECUNDARIA DE LAS INSTITUCIONES EDUCATIVAS ESTATALES DE LA UGEL 07 Y NIVEL DE PREPARACIÓN DEL DOCENTE PARA ABORDARLO
}

Mg. Sara M. Sáenz Chaparro Docente de la UMCH

Mg. José R. Vergara Díaz Docente de la UMCH

\section{Resumen}

El fenómeno del bullying es una problemática compleja que comprende no solo a los estudiantes que lo practican o padecen, sino también a los docentes que interactúan permanentemente en las aulas y en el centro educativo. Este trabajo buscó indagar sobre la presencia de bullying en los estudiantes del nivel secundario y la preparación docente para abordarlo. El diseño de investigación es descriptivo comparativo, la muestra fue de 789 estudiantes y de 40 docentes, todos ellos provenientes de centros educativos estatales de la UGEL 07. Los resultados encontrados señalan una prevalencia de bullying de $26,4 \%$ y un $22,5 \%$ de docentes que conocen y utilizan estrategias adecuadas para abordarlo. Se halló diferencias significativas en la prevalencia de bullying según grado de estudios; según género solo en tres dimensiones: coacción, agresiones e intimidación-amenazas siendo la media más alta en los varones. No se halló diferencias significativas en el abordaje del bullying por parte de los docentes según género, edad, tiempo de servicio y grado académico; por último no se encontraron diferencias en los puntajes de bullying de los estudiantes con docentes preparados y poco preparados. Estos resultados muestran que las actuaciones de los docentes, en ambos grupos, son limitadas para afrontar esta situación.

Palabras claves: bullying, abordaje, preparación docente. 


\section{Planteamiento del problema}

\section{I.I Descripción del problema}

Para todos, la violencia es una realidad que preocupa cada vez más. El sistema educativo no es ajeno a esta problemática, existen evidencias del maltrato físico y psicológico contra algunos estudiantes, víctimas del abuso y agresividad de sus compañeros, sea por su lugar de procedencia, por su buen rendimiento académico, por provenir de otro centro educativo, distrito u otras causas; todo maestro ha sido testigo presencial por lo menos una vez de este hecho. Por otro lado, en las investigaciones realizadas en el Perú por la Comisión Nacional para el Desarrollo y Vida sin Drogas (DEVIDA, 20 I3) a nivel nacional, se reporta que un porcentaje (38,7\%) del total de escolares de secundaria son víctimas de agresiones, hallando una asociación positiva entre el consumo de drogas y el bullying. Asimismo, se reportan como modalidades más frecuentes: apodos, insultos, difamaciones, ocultamiento, robo o rotura de cosas, ignorar, excluir, discriminar (DEVIDA, 2007; Oliveros \& Barrientos, 2007; Coicca, 2010).

En el trabajo con docentes se observa falta de capacidad para afrontar este tipo de problema. Las medidas que suelen aplicar son la llamada de atención y la expulsión de la clase, complementada con la citación a los padres. Se constata también, que si la falta es grave, se aplica la sanción determinada por el reglamento escolar y cuando la conducta inadecuada persiste, se condiciona su matrícula y/o es invitado a retirarse del colegio.

Por ello la presente investigación pretende no solo caracterizar el bullying en los estudiantes del nivel secundario de Instituciones educativas estatales de la UGEL 07 sino además hallar el nivel de preparación de los docentes tutores para abordarlo.

\section{I.2 Formulación del problema}

\section{Problema general}

La presente investigación plantea como problema: ¿Cuál es la prevalencia de bullying en estudiantes del nivel secundario de Instituciones educativas estatales de la UGEL 07 y el nivel de preparación de sus docentes para abordarlo?

\section{I.3 Justificación}

La presente investigación reviste actualidad y es de interés no solo educativo, sino público; en primer lugar, por la existencia de datos y estudios rigurosos sobre el estado de las relaciones interpersonales conflictivas dentro de la escuela, específicamente el bullying o acoso escolar cuya preocupación se debe a qué afecta la buena convivencia escolar. Los datos consultados son resultado de las investigaciones sobre abuso entre 
alumnos realizadas en países como: España, Noruega, Reino Unido, Estados Unidos, Brasil, entre otros (Hernández y Cárdenas, 20 I0). En el Perú las investigaciones son limitadas.

Su actualidad se constata en los medios de comunicación que tratan el tema y alertan a la sociedad sobre sus consecuencias: suicidio adolescente, por ejemplo. El interés por este problema se hace evidente en la percepción de los profesores y en las informaciones que la prensa escrita, radial y televisiva hace sobre la incidencia de casos de bullying, en una diversidad de instituciones educativas.

Existe pues, la necesidad de atender las relaciones entre pares con mayor profundidad y buscar los instrumentos adecuados para prevenir y/o abordar el bullying en el ámbito escolar. Por esta razón, interesa conocer la prevalencia del bullying y proporcionar datos sobre su detección, seguimiento y control en las instituciones educativas estatales.

Por otro lado, interesa determinar cuál es el nivel de preparación del docente para abordarlo. Los aportes de la presente investigación, benefician a la comunidad educativa y a la sociedad en general, porque proporciona datos sobre el estado de preparación docente ante esta problemática. Por esta razón, la evaluación rigurosa y científica de estos datos resulta fundamental para proponer y/o contrastar la efectividad de las acciones, planes y programas que se implementen como medida para controlar el acoso y la violencia en las aulas no solo por parte de los docentes sino además, por las Instituciones educativas y el Ministerio de Educación.

Finalmente, la presente investigación proporciona un instrumento que medirá el nivel de preparación docente para entender y abordar la problemática de la violencia y el acoso escolar.

\section{Objetivo General}

Determinar la prevalencia de bullying en los estudiantes de secundaria de Instituciones Educativas de la UGEL 07 y el nivel de preparación de sus docentes para abordarlo.

\section{Objetivos específicos:}

I) Determinar la prevalencia de bullying en alumnos de secundaria de las Instituciones Educativas de la UGEL 07.

2) Comparar la media del bullying según el género en alumnos de secundaria de las Instituciones Educativas de la UGEL 07. 
3) Comparar la media del bullying según el grado de estudios en alumnos de secundaria de las Instituciones Educativas de la UGEL 07.

4) Determinar el nivel de preparación docente frente al bullying en las Instituciones Educativas de secundaria de la UGEL 07.

5) Comparar el nivel de preparación docente frente a bullying según género en las Instituciones Educativas de secundaria de la UGEL 07.

6) Comparar el nivel de preparación docente frente a bullying según edad en las Instituciones Educativas de secundaria de la UGEL 07.

7) Comparar el nivel de preparación docente frente a bullying según tiempo de servicio en las Instituciones Educativas de secundaria de la UGEL 07.

8) Comparar el nivel de preparación docente frente a bullying según grado académico en las Instituciones Educativas de secundaria de la UGEL 07.

9) Comparar la media del bullying en estudiantes de secundaria con respecto al nivel de preparación para abordarlo de sus docentes tutores.

\section{Metodología}

\section{I Diseño de investigación}

Según el enfoque de Hernández, Fernández y Baptista (2010), la presente investigación es no experimental, ya que no existe manipulación activa de alguna variable. Además se trata de un diseño transversal, ya que se busca describir una realidad en un momento de tiempo siendo descriptivo y comparativo. Es descriptivo en vista que el estudio tiene como propósito describir la realidad que se observa en cada variable por separado, es comparativo porque tiene como propósito comparar variables o conceptos.

\subsection{Variables a comparar}

\section{Bullying}

Definición conceptual.- El hostigamiento o acoso escolar bullying, es cualquier forma de maltrato psicológico, verbal o físico producido entre escolares de manera reiterada a lo largo de un tiempo determinado Sanmartín (2007).

Definición operacional.-El bullying se evidencia con la puntuación obtenida por un alumno luego de aplicar el instrumento AUTOTEST -Cisneros de Piñuel y Oñate (2007) sobre acoso escolar. 


\section{Preparación Docente para abordar el bullying}

Definición conceptual.- Consiste en las capacidades y destrezas que poseen los docentes, en materia de conocimiento, estrategias y actitud frente al abordaje del problema de acoso escolar o bullying.

Definición operacional.- El resultado obtenido al aplicar el cuestionario a los docentes de los alumnos a los cuales se les aplicó el Autotest Cisneros sobre acoso escolar, referido a la preparación y estrategias de los docentes para abordar el bullying, luego de elaborar el instrumento PROF-BULL y validarlo.

Variables que hay que controlar: Sexo de los alumnos, sexo de los docentes, grado de estudios de los alumnos, edad de los docentes y tiempo de servicio.

\subsection{Población y muestra}

La población está conformada por 28487 alumnos del $1 .^{\circ}$ a $5 .^{\circ}$ grado y aproximadamente $\mid 537$ docentes del nivel secundario de estos grados de las 36 Instituciones Educativas Estatales de Educación secundaria que pertenecen a la jurisdicción de la UGEL 07.

La muestra estuvo compuesta por 789 casos del $1 .^{\circ}$ al $5 .^{\circ}$ grado de educación secundaria de EBR del turno mañana y tarde de Instituciones Educativas de la UGEL 07 y 40 docentes tutores de los grados de los estudiantes encuestados, después de la eliminación de aquellos docentes tutores que no pasaron el test de deseabilidad social con sus respectivos alumnos.

Para la selección de la Institución Educativa se tuvo en cuenta los colegios con mayor población específicamente aquellos que tienen más de 900 alumnos

Distribución de la muestra por institución educativa de la UGEL 07 Instituciones educativas

\begin{tabular}{|lcc|}
\hline & Frecuencia & Porcentaje \\
Jorge Chávez & 90 & 11.4 \\
José Olaya Balandra & 112 & 14.2 \\
Los precursores 7086 & 92 & 11.7 \\
Los próceres & 123 & 15.6 \\
Manuel Gonzales Prada & 41 & 5.2 \\
Mateo Pumacahua & 90 & 11.4 \\
Mercedes Indacochea & 117 & 14.8 \\
San pedro de Chorrillos & 37 & 4.7 \\
Santa Rosa 6094 & 87 & 11.0 \\
Total & 789 & 100.0 \\
\hline
\end{tabular}


La muestra fue distribuida en forma proporcional en las cinco secciones de cada centro educativo seleccionado, a continuación las variables demográficas:

\section{De los estudiantes}

\begin{tabular}{|l|l|c|}
\hline Género estudiante & M & $50 \%$ \\
\hline Grado del estudiante & F & $50 \%$ \\
\hline & $1^{\circ}$ & $22,8 \%$ \\
\hline $2 .^{\circ}$ & $17,2 \%$ \\
\hline $3 .^{\circ}$ & $19,9 \%$ \\
\hline $4^{\circ}$ & $16,5 \%$ \\
\hline & $5^{\circ}$ & $23,6 \%$ \\
\hline
\end{tabular}

\section{De los docentes}

\begin{tabular}{|l|l|l|}
\hline Género docente & M & $27,5 \%$ \\
\hline \multirow{2}{*}{ Edad } & F & $72,5 \%$ \\
\hline & $26-30$ & $10,0 \%$ \\
\hline Grado académico & $31-39$ & $30,0 \%$ \\
\hline & 40 a más & $60,0 \%$ \\
\hline & 11 a más & $70,0 \%$ \\
\hline & Licenciado & $75,0 \%$ \\
\hline & Magister & $25,0 \%$ \\
\hline
\end{tabular}

\subsection{Instrumentos}

Para el desarrollo de esta investigación se emplearon los siguientes instrumentos:

Autotest Cisneros (Cuestionario Individual sobre Psicoterror, Negación, Estigmatización y Rechazo en Organizaciones Sociales)

Este instrumento fue creado en España por Iñaki Piñuel y Araceli Oñate en 2005, a través del Instituto de Innovación Educativa y Desarrollo Directivo (IEDI). Fue adaptado por los investigadores de este estudio en marzo del 201 I.

Esta escala está diseñada para evaluar el Índice Global de Acoso Escolar y también permite hacer un perfil sobre las modalidades más frecuentes de acoso escolar o bullying.

La validez de contenido se realizó por juicio de expertos, adaptándose los ítems 23 y 43. La validez de constructo por el método de análisis de ítems, en el cual no se eliminó ningún ítem. La confiabilidad se halló con el Alfa de Cronbach cuyo valor fue de .948 , demostrando alto nivel de confiabilidad. 


\section{Se divide en 10 subescalas:}

Índice global de Acoso Escolar; Intensidad de Acoso; Desprecio-Ridiculización; Coacción; Restricción-Comunicación; Agresiones; Intimidación-Amenazas; Exclusión- Bloqueo Social; Hostigamiento Verbal; Robos. Consta de un total de 50 ítems.

\section{Prof-bull}

Este instrumento fue elaborado para la presente investigación. Está diseñado para medir la preparación del docente para abordar el bullying. Proporciona un perfil sobre el tipo de formación recibida y las acciones más frecuentes que realiza el docente en caso de detectar bullying.

La validez de contenido se realizó por juicio de expertos, revisando la redacción y las dimensiones. La validez de constructo con el método de análisis de ítems, eliminándose 8 ítems. La confiabilidad según el Alfa de Cronbach fue de .915, lo que indica una muy buena confiabilidad.

\section{Se divide en 6 subescalas}

Índice Global de preparación docente para abordar el bullying; Noción del problema; Capacidad de identificar el problema; Acciones de abordaje del problema; Autoevaluación de la propia capacidad frente al problema; Deseabilidad Social. Consta de un total de 48 ítems.

Para establecer el menor o mayor grado de acoso escolar se ha obtenido los baremos según la muestra piloto de 296 alumnos de Instituciones Estatales de la UGEL 07.

Se considera a los puntajes que están dentro de los percentiles de I a 24, valor bajo; de 25 a 74, valor promedio; de 75 a 94, valor alto; de 95 a 100, valor muy alto.

El nivel bajo indica que no hay bullying, el nivel medio son conductas que suceden pocas veces dentro de un nivel normal donde se dan comportamientos en el aula con las distintas manifestaciones de agresividad pero que no se considera bullying.

El nivel alto son conductas más frecuentes en el aula con alto riesgo de ser bullying y el nivel muy alto se considera bullying severo y afecta gravemente al estudiante víctima.

Para establecer el menor o mayor grado de preparación del docente para abordar el bullying o acoso escolar se ha obtenido los baremos según la muestra de 40 profesores de Instituciones Estatales de la UGEL 07. 
Se han considerado los puntajes que están dentro de los percentiles de I a 24, valor bajo; de 25 a 74, valor promedio; de 75 a 94, valor alto y de 95 a I00, valor muy alto.

El nivel bajo se considera a los profesores con muy poca preparación para abordar el bullying. El nivel medio se considera a los profesores que tiene preparación para abordar situaciones de violencia o agresión comunes que se dan en el aula y emplean las estrategias que comúnmente se usan en los colegios. Llamar a los padres, hablar con ellos, aconsejar y orientar en el aula, derivar a disciplina.

El nivel alto son los docentes que tienen una mejor preparación sobre el bullying y las estrategias que utilizan les resulta a veces efectiva.

El nivel muy alto de preparación son aquellos docentes que no solo conocen este fenómeno sino que además emplean estrategias efectivas en el abordaje de los mismos.

\section{Resultados}

En la figura I se muestra los porcentajes de bullying de los resultados globales de la escala de acoso escolar, se evidencia la existencia de bullying en un $26,4 \%$ en los porcentajes acumulados del nivel alto y muy alto de la muestra de estudio, de la cual un 4,6\% se encuentra en un bullying severo. Un 57.2\% en un nivel medio lo que indica un nivel normal, es decir existen relaciones de agresión pero que no son consideradas bullying y un 16,5\% un nivel bajo donde no hay bullying ni otro de tipo de violencia o agresión. Con respecto a las modalidades más frecuentes del bullying se ha encontrado que son: Le llaman por apodo, no le hablan, se ríen cuando se equivoca, no le dejan jugar con ellos, le insultan, roban sus cosas, cuentan mentiras acerca de ellos, no le dejan hablar, le odian sin razón, le acusan de cosas que no ha dicho y se burlan de su apariencia física.

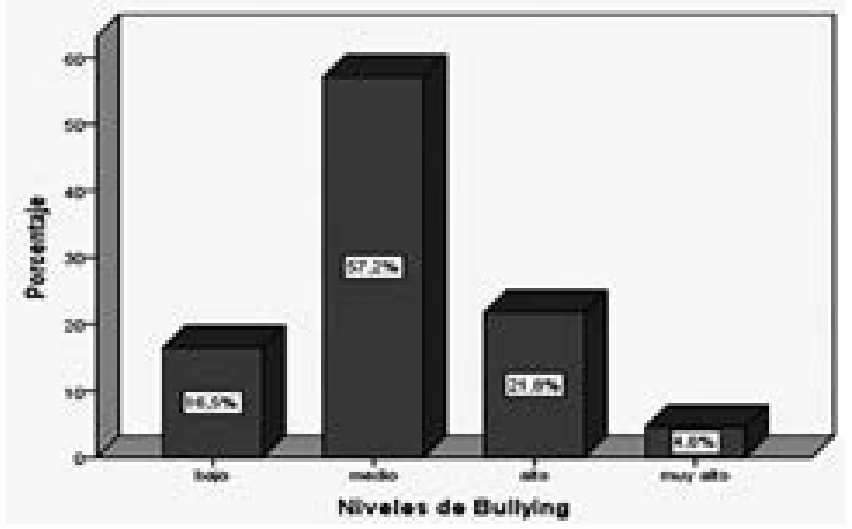

Fig. I. Porcentajes de bullying 
En la figura 2 se observa los niveles acumulados de intensidad de bullying en las categorías alto y muy alto un $27,9 \%$ de la muestra de estudiantes que es víctima de frecuente maltrato escolar de la cual un 3,7\% se encuentra en los límites muy altos, un $17,4 \%$ sufren algún tipo de maltrato no muy frecuente dentro de lo normal y un $54.8 \%$ manifiesta no ser víctima de bullying.

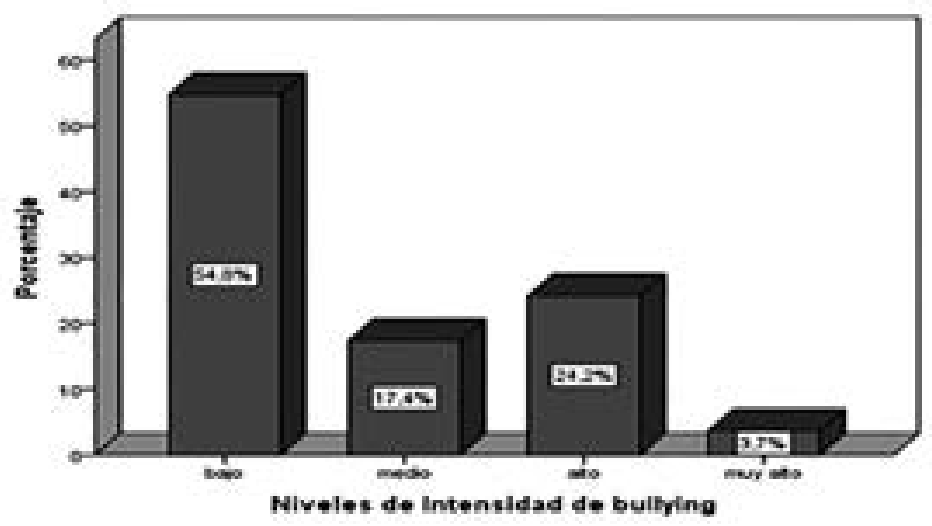

Figura 2. Intensidad de bullying

En la tabla se observa que la media para el índice global se ubica en normal promedio, la media para intensidad de acoso, Coacción, Intimidación-amenazas se ubica en un nivel alto, la media de Desprecio-Ridiculización, Restricción- Comunicación y Exclusión-Bloqueo Social en casi alto, agresiones, hostigamiento verbal y Robos en un nivel normal promedio.

Análisis de la variable bullying según sus dimensiones

\begin{tabular}{l|c|c|}
\hline Dimensiones & Media & Nivel \\
\hline Índice global & 63.65 & Normal \\
\hline Intensidad de acoso & 1.93 & Alto \\
Desprecio-ridiculización & 22.88 & Casi alto \\
Coacción & 9.07 & Alto \\
Restricción -Comunicación & 7.05 & Casi alto \\
Agresiones & 8.98 & Normal \\
Intimidación-amenazas & 11.25 & Alto \\
Exclusión-bloqueo social & 10.77 & Casi alto \\
Hostigamiento verbal & 17.27 & Normal \\
Robos & 5.18 & Normal
\end{tabular}


Comparación de las dimensiones de bullying con respecto al género

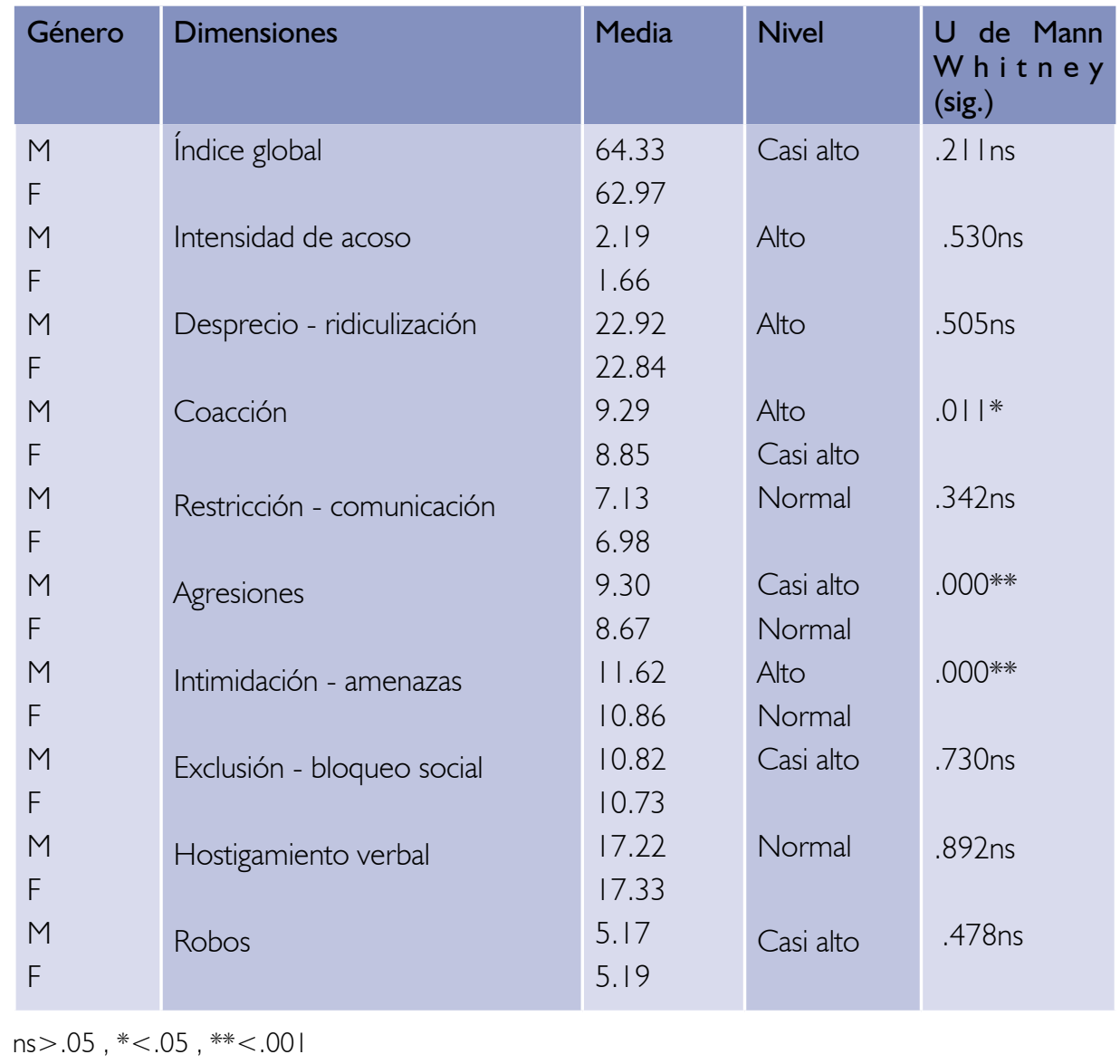

Comparación de las dimensiones de bullying con respecto al grado de estudios

\begin{tabular}{|c|c|c|c|c|}
\hline Grado de estudios & Dimensiones & Media & Nivel & Kruskal-Wallis (sig.) \\
\hline $\begin{array}{l}1 .{ }^{\circ} \\
2 .{ }^{\circ} \\
3 .{ }^{\circ} \\
4 .^{\circ} \\
5 .^{\circ}\end{array}$ & Índice global & $\begin{array}{l}67.23 \\
64.51 \\
61.89 \\
62.92 \\
61.56\end{array}$ & $\begin{array}{l}\text { Casi alto } \\
\text { Normal }\end{array}$ & $.000 * *$ \\
\hline $\begin{array}{l}1 .^{\circ} \\
2 .^{\circ} \\
3 .^{\circ} \\
4 .^{\circ} \\
5 .^{\circ}\end{array}$ & $\begin{array}{l}\text { Intensidad de } \\
\text { acoso }\end{array}$ & $\begin{array}{l}3.08 \\
2.09 \\
1.38 \\
1.73 \\
1.29\end{array}$ & $\begin{array}{l}\text { Alto } \\
\text { Casi alto }\end{array}$ & $.000 * *$ \\
\hline
\end{tabular}




\begin{tabular}{|c|c|c|c|}
\hline $\begin{array}{l}1 .{ }^{\circ} \\
2 .^{\circ} \\
3 .^{\circ} \\
4 .^{\circ} \\
5 .^{\circ}\end{array}$ & $\begin{array}{l}\text { Restricción } \\
\text { comunicación } \\
\text { Exclusión - bloqueo } \\
\text { social }\end{array}$ & $\begin{array}{l}\text { Casi alto } \\
\text { Normal }\end{array}$ & $.000 * *$ \\
\hline $\begin{array}{l}1 .^{\circ} \\
2 .^{\circ} \\
3 .^{\circ} \\
4 .^{\circ} \\
5 .^{\circ}\end{array}$ & $\begin{array}{l}\text { Desprecio - ridiculización } \\
\text { Coacción } \\
\text { Intimidación y amenazas } \\
\text { Hostigamiento verbal }\end{array}$ & $\begin{array}{l}\text { Alto } \\
\text { Casi alto } \\
\text { Normal }\end{array}$ & $<.05^{*}$ \\
\hline
\end{tabular}

Análisis de la variable preparación del docente para abordar el bullying

\begin{tabular}{l|l|l}
\hline Dimensiones & Media & Nivel \\
\hline Índice global & 84.78 & Normal \\
Noción del problema & 16.00 & Casi alto \\
Capacidad de identificar & 30.75 & Normal \\
Acciones de abordaje & 30.05 & Normal \\
Autoevaluación de & & \\
la propia capacidad & 7.98 &
\end{tabular}

Comparación del nivel de preparación docente frente al bullying según género, edad, tiempo de servicio y grado de estudios

\begin{tabular}{|c|c|c|c|c|c|c|}
\hline Variable & & Dimensión & Media & Nivel & (sig.) & Estadístico \\
\hline \multirow[t]{2}{*}{ Género } & $M$ & \multirow{10}{*}{ Índice Global } & 86,36 & Casi alto & $.463 n s$ & T de student \\
\hline & F & & 84,17 & Promedio & & \\
\hline \multirow[t]{3}{*}{ Edad } & $26-30$ & & 86,00 & Casi alto & & \\
\hline & $31-39$ & & 85,67 & Promedio & & \\
\hline & 40 a más & & 84,13 & Promedio & & \\
\hline \multirow{4}{*}{$\begin{array}{l}\text { Tiempo de } \\
\text { servicio }\end{array}$} & I a 3 & & 83,50 & Promedio & $.930 \mathrm{~ns}$ & Kruskal Wallis \\
\hline & 4 a 6 & & 84,00 & & & \\
\hline & 7 a 10 & & 83,00 & & & \\
\hline & II a más & & 85,36 & & & \\
\hline $\begin{array}{l}\text { Grado } \\
\text { acadé- } \\
\text { mico }\end{array}$ & $\begin{array}{l}\text { Licenciado } \\
\text { Magister }\end{array}$ & & $\begin{array}{l}84,97 \\
84,20\end{array}$ & Promedio & $.950 \mathrm{~ns}$ & Kruskal Wallis \\
\hline
\end{tabular}

ns $>.05, *<.05, * *<.001$ 
Resultados obtenidos de la comparación del puntaje de bullying con relación al nivel de preparación de sus docentes para abordarlo:

Índice Global de bullying

\begin{tabular}{|l|l|l|}
\hline \multicolumn{1}{|c|}{ Índice global de profesores } & \multicolumn{2}{c|}{ Media } \\
\hline Menor preparación & 63.00 & Normal \\
\hline Mayor preparación & 65.74 & Casi alto \\
\hline Total & \multicolumn{1}{|c|}{ Índice global de acoso } \\
\hline & & 51404.000 \\
\hline U de Mann-Whitney & & -1.940 \\
\hline Z & & -1.940 \\
\hline Sig asintótica(bilateral) & \\
\hline
\end{tabular}

\section{Discusión de resultados}

El presente estudio determinó un $26,4 \%$ de prevalencia de bullying en la muestra estudiada, este resultado se relaciona cercanamente con las investigaciones de Cassiani et al. (20 I I), Becerra et al. (2008), Oñate y Piñuel (2007) y Paredes et al. (2006). En cambio se aleja de los resultados encontrados en la investigación de Oliveros et al. (2009), debido a que se realizó en regiones consideradas escenarios de violencia política y de los resultados obtenidos por Coicca (20 I0) considerando que este estudio se aplicó en un solo colegio ubicado en una zona de alto riesgo en el distrito de Comas, razón por la cual estos resultados no coinciden con la presente investigación.

Este resultado indica que el bullying es un fenómeno frecuente en los estudiantes de secundaria en cualquier Institución Escolar de la UGEL 07 y que existen estudiantes que se encuentran en el nivel muy alto de bullying por lo que se presentan problemas de salud y es necesario tomar medidas urgentes y efectivas para evitar el daño que este ocasiona. En lo referente a las modalidades más frecuentes de bullying hallados en esta investigación, se puede afirmar que predominan las siguientes: "me llaman por apodo" (14,7\%), "no me hablan" (12,0\%), "se ríen de mi cuando me equivoco" (7,5\%), "no me dejan jugar con ellos" (7,4\%), "me insultan" (6,7\%), "roban mis cosas" (6,6\%), "cuentan mentiras acerca de mí" (6,2\%). Estas manifestaciones de bullying coinciden con los resultados de DEVIDA (2007), Oliveros y Barrientos (2007), Coicca (20 I 0) y Ávila -Toscano et al. (20 I0), que hallaron que la agresión verbal era la que predominaba. 
Los datos indican que no existen diferencias significativas en el género con respecto al índice global, lo cual coincide con el estudio de Avilés (2006) y Ávila-Toscano et al. (2010) que equiparan ambos sexos en la participación de actos de maltrato, así como en su participación como víctima. Esto significa que es un fenómeno mucho más generalizado y que va cobrando participación de las mujeres implicadas en estos actos de violencia. Sin embargo se halló diferencia en tres dimensiones: Coacción, Agresión e Intimidación -Amenazas, presentando los varones mayor nivel de acoso en estas áreas. Estos resultados coinciden con los estudios de (Ávila-Toscano et al., 20 I0; García, 2007) que señalan que las diferencias significativas se expresan en las modalidades de intimidación y que son los varones los que más participan en la dinámica del bullying.

Este hallazgo puede explicarse por el tipo de intimidación que se ejerce según sexo, considerando que los varones ejercen más la agresión verbal y física y las mujeres formas de hostigamiento más sutiles, conforme a las características propias de cada género y a sus formas de socialización (citado por García, 2007).

En el análisis de los resultados de la prevalencia del bullying según el grado de estudios, se hallaron diferencias significativas en las siguientes dimensiones: Índice global, Intensidad de Acoso, Restricción-comunicación, Exclusión-bloqueo social, Desprecio-Ridiculización, Coacción, Intimidación-Amenazas y Hostigamiento verbal, obteniendo el primer año de secundaria la media más alta, seguido del segundo año de secundaria; estos resultados coinciden con las investigaciones de Avilés (2002, 2006), Oñate y Piñuel $(2005,2007)$ que hallaron los mayores índices de acoso escolar en el primer y segundo año de la ESO.

Hay consenso en que el número agresores como victimizados desciende con la edad y grado de estudios (Ávila-Toscano et al., 20 I0; Chávez, Garay, Hamasaki, 2013). Esto se debe a que el grado y la edad coinciden con los cambios que se producen en las distintas etapas de la adolescencia, tales como los procesos de maduración y socialización, la formación de grupos, el desarrollo de la personalidad y sexualidad que varían conforme avanza la edad (Avilés, 2006; Ramos 2008; Ávila-Toscano et al., 20 I0; Méndez y Cerezo, 20 I0; Rincón, 20I I) influenciados por factores familiares tales como: crianza de los padres, dinámica de las relaciones familiares, condición social, cultural, económica y del entorno: tipo de barrio, grupos sociales, medios de comunicación, modos de convivencia en la escuela (Avilés, 2006; Ortega 20 I0).

Los resultados obtenidos sobre la preparación del docente para abordar el bullying permiten determinar que el 5\% está altamente capacitado para abordar el bullying y las estrategias que utiliza son efectivas; el 17,5\% tiene una preparación adecuada que le permite identificar y actuar en caso de bullying con efectividad. Esto implica 
que el 22,5\% tiene una buena preparación, el 47,5\% utiliza mecanismos conocidos en la Institución que buscan resolver casos de violencia, agresión, disrupción en el aula, indisciplina de manera general, no específicamente bullying, siendo un 30\% los docentes que no tienen la preparación suficiente para abordar este fenómeno. Estos resultados coinciden con los hallados por Elizalde (2010) quien considera que hay una falta de utilización de estrategias en el manejo del bullying por los profesores.

Con respecto a las actuaciones más frecuentes de los docentes para abordar el bullying en cuanto a identificar el problema se encontró que el docente conoce los apodos, está atento a las incidencias que ocurren en el aula y el diálogo permanente con los estudiantes. Así también en las acciones de intervención del docente se reconocen el diálogo individual con cada uno de los estudiantes implicados, la orientación al aula para prevenir nuevos incidentes, la derivación de los casos al Consejo de tutores, Normas y convivencia, dpto. Psicopedagógico y Dirección, por último diálogo con los padres. Estos resultados van en la línea de otros estudios (Serrano, 20 I3; Ochoa \& Peiró, 20 I0; Oliveros, 2008) quienes también hallaron los mismos indicadores, de acuerdo con Oliveros y Barrientos (2007) manifiestan que los docentes utilizan las medidas convencionales de la escuela en caso de bullying lo cual evidencia que hay un manejo inadecuado del bullying .

Es evidente que los docentes reaccionan ante el acoso con castigo (sanción, según las normas) a los agresores, otros intervienen para cortar este tipo de situaciones y un grupo no se entera de la situación, esta es la forma como resuelven los profesores el acoso, Garaigordobil y Oñederra (2009). Esto explicaría el resultado de este estudio con respecto a la preparación del docente para abordar el problema de bullying , todo indica que los docentes no están preparados y las que estrategias que utilizan no son las más adecuadas.

Con respecto a los resultados hallados en la comparación del nivel de preparación docente frente al bullying según género, no se hallaron diferencias significativas en todas las escalas. Estos resultados coinciden con Meléndrez y Miranda (2012) y Gómez et al (2008) quienes encontraron que no existe una relación causal entre las competencias de los maestros y su género, es decir, que el género no influye en el nivel de desempeño docente. García, Orellana, Yanac, Herrera, Campos, Campos, Macasana, Orellana y Fernandini (20I3), no encuentran diferencias significativas en casi todos los ítems de los factores predictores de bullying con respecto al género, sólo en algunos aspectos particulares. Ararteko (2006), estudia la percepción de los docentes con respecto al bullying y relaciona algún atributo del desempeño que los docentes valoran la necesidad de formación en competencias para actuar en episodios de bullying . 
En cuanto a los resultados de comparación de la edad del docente con su preparación para abordarlo, se encontró que no existen diferencias significativas en todas las dimensiones, esto debido a que los modos de enfrentar este fenómeno del bullying en los diferentes rangos de edades tienen características similares o en todo caso se equiparan en cuanto al resultado de sus acciones.

En la comparación de la preparación docente para abordar el bullying según tiempo de servicio, no se encontraron diferencias significativas en todas las dimensiones. Estos resultados coinciden con los estudios de Gómez et al (2008), quienes señalan de acuerdo a la percepción de los estudiantes y las variables contratación, grado académico, antigüedad, y género no existen diferencias significativas $(p>0.05)$ con el desempeño docente, también con Fernández et al. (2006), que señalan que la autopercepción de la capacidad para afrontar el bullying, no difiere según el ciclo educativo al que pertenece ni según la experiencia docente que posee. Esto se explicaría por la praxis de abordaje docente, en situaciones que no son necesariamente bullying , en el quehacer diario de las clases. En cuanto a las acciones de abordaje se ubicaron en un promedio normal lo que significa que, toman medidas conocidas para abordar las conductas violentas, pero no necesariamente bullying , logrando cierta efectividad.

En la comparación de grado académico no se hallaron diferencias significativas. Meléndrez et al. (2012) y Gómez et al (2008), señalan que no existe diferencia significativa (0.05) en el grado académico con el desempeño docente, considerando que lo que hace a un profesional es la trayectoria laboral que el docente posea, para la transmisión de conocimientos. De acuerdo al presente estudio, se concluye que el grado académico, no influye en el nivel de preparación que poseen los docentes para abordar el bullying .

En el análisis de la relación del puntaje de bullying en estudiantes de secundaria y el nivel de preparación de sus docentes tutores para abordarlo se encontró que los valores de las medias son cercanos, no existiendo diferencia significativa; estos resultados muestran que las actuaciones de los docentes son limitadas para afrontar estas situaciones. De esto se deriva que en las Instituciones Educativas donde se presentan más casos de bullying algunos docentes incrementan su nivel de preparación para abordarlo; en cambio donde la manifestación de bullying es menor, se desentienden de su preparación. Se puede concluir que la preparación del docente para abordar el bullying es necesaria e importante aunque no determinante para lograr una intervención que disminuya este fenómeno. 
Por otra parte, el fenómeno de acoso escolar es un problema complicado y multifactorial, en el que median desde las características personales tanto de docentes como de los estudiantes implicados hasta la expresión de factores relativamente independientes de la escuela, como los problemas personales, los trastornos de relación, la influencia del grupo de amigos o la familia (Palomero, 200 I). Asimismo, el clima escolar, tanto del grupo, como del aula y del centro, las relaciones entre los agentes educativos, el método de crianza, la escuela, el barrio, los medios de comunicación y otros.

Para prevenir la violencia escolar Díaz - Aguado nos dice que es preciso adoptar una perspectiva ecológica que permita conceptualizar las condiciones de riesgo y de protección en función de la interacción entre el individuo y el entorno a distintos niveles, prestando una especial atención a la forma de estructurar las actividades escolares, así como a la colaboración de la escuela con las familias y con el resto de la sociedad.

\section{Conclusiones}

- Se halló una prevalencia de bullying de 26,4\% y un nivel de preparación docente de 22,5\% en la muestra estudiada, confirmando la hipótesis de la investigación.

- No existen diferencias significativas en la prevalencia del bullying según género en las Instituciones Educativas de la UGEL 07 en el nivel de secundaria en la escala global, sólo se halló en tres dimensiones: Coacción, Agresiones e Intimidación amenazas siendo la media más alta en los varones.

- Existen diferencias significativas en la prevalencia del bullying según grado de estudios en las Instituciones Educativas de la UGEL 07 en el nivel de secundaria. Las investigaciones señalan que en los primeros grados de educación secundaria existe mayor prevalencia de bullying, hecho que confirma la presente investigación.

- No existen diferencias significativas en la preparación docente para abordar el bullying, según género, en las Instituciones Educativas de la UGEL 07 en el nivel de secundaria, situación que es consistente con otras investigaciones.

- No existen diferencias significativas en la preparación docente para abordar el bullying según edad en las Instituciones Educativas de la UGEL 07 en el nivel de secundaria.

- No existen diferencias significativas en la preparación docente para abordar el bullying según tiempo de servicio del docente en las Instituciones Educativas de la UGEL 07 en el nivel de secundaria. 
- No existen diferencias significativas en la preparación docente para abordar el bullying según grado académico del docente en las Instituciones Educativas de la UGEL 07 en el nivel de secundaria.

- No existen diferencias significativas en los puntajes del bullying en los estudiantes de secundaria de la UGEL 07 con respecto al nivel de preparación que, los docentes tutores, tienen para abordarlo.

\section{7.- Recomendaciones}

- Ampliar el campo de investigación hacia otras UGEL que conforman Lima Metropolitana a fin de obtener una visión global de esta realidad.

- Recomendar la coordinación de eventos de carácter preventivo en las escuelas públicas y privadas a fin de abordar de manera integral y multidisciplinaria este fenómeno.

- Ampliar el acceso a los colegios no solo de psicólogos educativos sino también de psicopedagogos.

- Ampliar el trabajo en red con Instituciones del Estado y las no gubernamentales, a través de proyectos a nivel de UGEL que garanticen la erradicación del bullying y con ello la buena convivencia en las Instituciones Educativas.

- Promover la aplicación de la ley en relación a la contratación de psicólogos educativos.

- Realizar estudios sobre la preparación que reciben los docentes, en su etapa formativa, en relación al abordaje del bullying.

- Revisar los programas de capacitación docente, relacionados al abordaje del bullying, con el fin de mejorar los contenidos y estrategias.

- Recomendar a los sectores que corresponda, la revisión y optimización de los programas de formación docente, específicamente en lo relacionado al abordaje del bullying.

- Sugerir a las Universidades y lugares de Formación Pedagógica la implementación en su malla curricular de cursos específicos relacionados al abordaje de bullying

- Recomendar a las Instancias Superiores: Municipios, Centros de Salud, Parroquias, Asociaciones, etc., fortalecer el trabajo con las familias, en cuanto a la educación en valores de los hijos, y los roles de padre y madre, en el marco de la paternidad responsable. 


\section{Referencias}

Alcazár, J. C., Bousoz, G., Losada, R., Losada M. A., Nazario, J.M. (2007). Acoso escolar: Desde la sensibilización social a una propuesta de intervención. Revista electrónica de Derecho, 4, I-6.Recuperado dehttp://dialnet.unirioja.es/ servlet/articulo?codigo $=2888550$

Alvarez L.,Alvarez D., González-Castro P., Nuñez J. C. y Gonzáles-Pienda, J. A. (2006). Evaluación de los comportamientos violentos en los centros educativos. Psicothema, I8(4), 686-695.

Ararteko (2006). Convivencia y Conflictos en los Centros Escolares. Recuperado de:

http://2 13.0.8. I 8/portal/Educantabria/RECURSOS/Materiales/Biblestinv/EuzKadi_ Convivencia_conflicto_CenEscolares_06.pdf

Avila-Toscano, J. H., Osorio, L., Cuello K., Cogollo, N. y Causado, K. (20l0). Conducta bullying y su relación con la edad, género y nivel de formación en adolescentes.Psicogente, I3(23), 13-26.

Avilés, J. M. (2009). Victimización percibida y Bullying. Boletín de Psicología, 95, 7-28.

Becerra, Flores y Vásquez (2008). Acoso escolar ( bullying) en Lima Metropolitana (Tesis de Licenciatura). Universidad Federico Villarreal. Lima. Perú.

Benites, L., Carozzo, J., Horna, V., Palomino, L.,Salgado, C., Uribe, C., Zapata, L. (20 I 2). Bullying y Convivencia en la Escuela. Lima: Observatorio sobre violencia y Convivencia en el aula.

Calvo, A. y Ballester, F. (2009). Acoso Escolar. Programas de Intervención. Madrid: Eos.

Casas, L. y Echandía, J. (20 I3). La experiencia laboral y el tipo de gestión en la identidad profesional docente en el distrito de Miraflores (Tesis de maestría). Universidad Marcelino Champagnat. Lima, Perú.

Cassiani, C., Gómez, J., Cubides, A. y Hernández, M.(20I I). Prevalencia de bullying y factores relacionados en estudiantes de bachillerato de una institución educativa de Cali, Colombia, 20 I I. Revista Salud Pública. 16 (I): I4-26.

Chapi, J. (20/2). Una revisión psicológica a las teorías de la agresividad. Revista electrónica de Psicología Iztacala, I 5(I ),81-92. 
Coicca, T. (2010). Bullying y Funcionalidad Familiar en una Institución Educativa del Distrito de Comas. (Tesis de Licenciatura) Universidad Universidad Nacional Federico Villarreal. Lima,Perú.

Craig, W. M., Henderson, K. y Murphy, J. G. (2000). Prospective teachers' attitudes toward bullying and victimization. School Psychology International,2I (I), 5-2I.

Cobo ,Py Tello,R (20 I I). Bullying: El acecho cotidiano en las escuelas. México: Limusa.

Defensor del Pueblo (2007). Violencia Escolar: El Maltrato entre iguales en la Educación Secundaria Obligatoria 1999-2006. Madrid: Defensor del Pueblo.

DEVIDA (2007). Estudio Nacional: Prevención y consumo de drogas en estudiantes secundarios. Recuperado de:http//www.devida.gob.pe/Documentación/ documentosdisponibles/IEstudio Regional Escolares Sec 2007.pdf

Díaz-Aguado, M. (2006) Convivencia escolar y prevención de la violencia. Madrid: Ministerio de Educación y Ciencia, CNICE.

Elizalde, A. (20।0). Estudio descriptivo de Estrategias de afrontamiento del bullying en el profesorado mexicano. Revista electrónica en Educational Psichology,8(I), 353- 372.

Estévez, E. (2005) Violencia, victimización y rechazo escolar en la adolescencia (Tesis doctoral). Universidad de Valencia. Valencia, España. Recuperado de: https:// www.uv.es/isis/estevez/tesisestevez.pdf

Garaigordobil, M., Oñederra, J. (2009). Acoso y violencia escolar en la comunidad autónoma del País Vasco. Psicothema,2I ( I), 83-89.

García, L. (2007). Diferencias de género y atribuciones causales en los protagonistas del bullying. Revista Magistri et doctores, 3, 87- 109.

García, L., Orellana, O.; Pomalaya, R.; Yanac, E; Orellana, D.; Sotelo, L.; Herrera, E; Sotelo, N.; Chavez, H. y Fernandini, P. (20l I). Intimidación entre iguales(bullying): Empatía e inadaptación social en participantes de bullying. Revista IIPSI , I 4 ( 2),27I - 276.

Gil-Verona,J., Pastor,J.,De paz, F.,Barboza, M.,Macías, J., Maniega, M., RamiGonzales,L., Boget, T., Picornell, I (2002).Psicobiología de las conducta agresivas. Anales de la Psicología 18 (2),293-303. 
Gómez, A., Gala, F.J., Lupiani, M., Bernalte, A., Miret, M.T., Lupiani, S. y

Barreto, M.C. (2007). El "bullying" y otras formas de violencia adolescente.

Cuadernos de Medicina Forense, 13, 165-177. Recuperado de:

http://scielo.isciii.es/pdf/cmf/n48-49/art05.pdf

Gómez, C. (20/4). Factores asociados a la violencia: Revisión y posibilidades de abordaje. Revistalberoamericanade Psicología:Cienciay Tecnología,7( I ), I I 5- 124.

Gómez, C., Elboj, C.,Luna, M. J., Puyal, E.,Sanagustín, M. V. y Sanz, A.(2007). Convivencia en los Centros Educativos. Módulo 4: convivencia profesores y alumnos. Zaragosa: GORFISA. 